\title{
There is nothing 'incidental' about unrelated findings
}

\section{"I suggest herein that the genomics research community replace the term 'incidental findings' with the term 'unrelated findings', given that there is nothing incidental about proving causality for any mutation."}

\section{KEYWORDS: ethics " exome sequencing " genetics = incidental $\approx$ unrelated finding whole-genome sequencing}

The specific topic for discussion in this editorial concerns the nature of 'unrelated findings', along with suggestions for how such issues should be handled in the future. In addition to focusing on highly penetrant Mendelian disorders [1,2], I am focusing my research on childhood-onset neuropsychiatric disorders, including attention deficit hyperactivity disorder (ADHD), obsessive compulsive disorder, Tourette's syndrome, developmental delay and mental retardation. After finishing clinical training in psychiatry, I moved to the state of Utah, USA in 2009, so that I could study large pedigrees for human genetics there, given that families tend to be large in Utah and the genealogy records of the Mormon Church are quite extensive. Utah has therefore been, and continues to be, a very fertile ground for human genetics research, with perhaps the most famous genetics finding involving the cloning of the breast cancer gene, BRCA1 [3]. In 2011, we published [1] and discussed elsewhere [2] our recent discovery in Utah of a new infantile lethal disease, dubbed Ogden syndrome, in honor of the city where the first family lives. I would like to discuss here a more recent project, in which we have had to struggle with what we have termed 'unrelated findings' coming out of a whole-exome sequencing project performed on one family in Utah.

In late 2009, we decided to apply exome sequencing to a complex disease, namely ADHD. Psychiatric genetics is a much harder problem than that of a fully penetrant infantile lethal syndrome, and our paper was one of the first (to my knowledge), explicitly discussing how we actively pursued and then handled an unrelated finding arising from our study [4]. In this instance, in addition to identifying rare variants that might contribute to $\mathrm{ADHD}$, we determined the genetic basis of an unrelated case of idiopathic hemolytic anemia. As these results were not Clinical Laboratory Improvement Amendments (CLIA) certified, I conveyed the anemia results to a physician of the research participant for validation and follow-up in a CLIA-certified environment. I grappled with the very difficult ethical and moral need to pursue the genetic basis of disorders unrelated to the primary research purpose, particularly when one finds out about such disorders after the sequencing has commenced. Hank Greely has argued forcefully that anyone participating in a genetics research study is protected by the human subjects research policies, including the ethical and moral need to pursue and return unrelated findings to such participants [5]. Given that I agree that the collection and sequencing of families falls squarely in the area of human subjects research, I have suggested here and elsewhere that researchers consider sequencing whole genomes in CLIAcertified laboratories going forward, either at Complete Genomics (CA, USA), Illumina (CA, USA) or elsewhere, depending on the quality and price [6]. I have also discussed the need for CLIA certification in terms of the moral need to return 'unrelated findings' that can emerge during such research $[4,6]$. I suggest herein that the genomics research community replace the term 'incidental findings' with the term 'unrelated findings', given that there is nothing incidental about proving causality for any mutation.

\section{An unrelated finding}

As the cost of next-generation sequencing continues to decrease exponentially, it is becoming both affordable and relatively easy for many laboratories, including those outside of large-scale sequencing centers, to perform exon capture, and eventually, whole-genome sequencing in

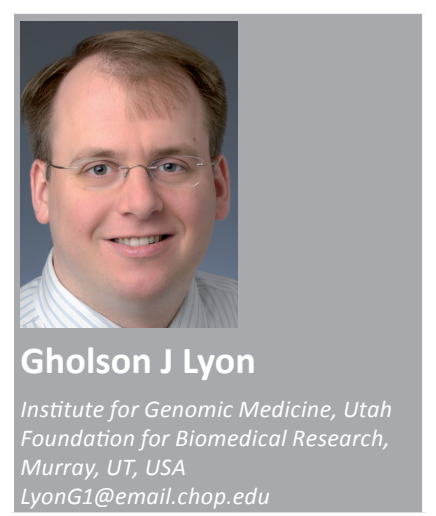

Future $\mathrm{fSS}$ 
selected individuals. This is already revolutionizing the discovery of the genetic basis of many disorders that had resisted conventional linkage mapping efforts [7-10]. As a result, there is now an ongoing avalanche of human genome sequencing results, emanating from many laboratories and from many different sequencing platforms. In 2009, I joined the institutional review board protocols at the University of Utah (UT, USA), which allowed me to collect research subjects for human genomics research. Over the course of the next year, along with my colleagues, I enrolled and consented many dozens of families for genetic research, focused primarily on simple Mendelian disorders and childhood-onset neuropsychiatric disorders.

During the course of research, I decided to perform exome sequencing on one family with a mother, father and two sons. As described in our paper, the father and two sons had a severe form of ADHD, with a very similar phenotype among them, including a similar response to a methylphenidate derivative in a double-blind placebo-controlled trial [4]. As we described in the paper, one of the sons informed me during a routine research visit that he had recently had a splenectomy, apparently resulting from a case of idiopathic hemolytic anemia. Given that I had already sequenced his exome, I felt an ethical and moral obligation to analyze the exome data to see if we could explain why he might have this anemia. After many filtering steps, we discovered that this person was a compound heterozygote for two mutations in PKLR, with both mutations having been seen one time before in combination with other mutations in separate individuals also with hemolytic anemia. However, there was no person reported in the literature possessing both mutations in this particular compound heterozygote state, so it was difficult to know causality, particularly with this person's unique genetic background and environmental influences (i.e., penetrance). Therefore, we performed blood and enzymatic activity assays to confirm that his anemia likely resulted from these mutations, and we published some of these data in our paper [4]. Unpublished testing also included biochemical affinity assays performed on packed red cell concentrates, prepared from the patient's blood (after removal of white cells and platelets), with results consistent with our conclusion that these mutations were causative for hemolytic anemia. This was an unrelated finding in the sense that our initial research purpose was to understand the genetic basis of the ADHD, but we discovered something unrelated to this purpose during our research.

There are many scientists and ethicists that are using the term 'incidental finding' to refer to unrelated findings arising from genomics research. This term, 'incidental finding' is mostly used in the radiology field, where highly trained radiologists look at a film or image and recognize something outside the range of 'normal' but unrelated to the clinical question, which they sometime refer to as an 'incidental' or 'coincidental finding' [11,12]. A PubMed search in November 2011 for the term 'incidental findings' returns references to a mind boggling 12,788 papers, of which 4027 are encompassed within the subsearch of 'incidental findings AND radiology'. On the other hand, a PubMed search for 'incidental findings AND genetics' returns 543 papers, a number far less than in radiology, but which reflects the growing use of this term in the genetics community. Obviously, this is only a crude measure, given that even a casual inspection of the titles and abstracts for many of these genetics papers reveals an obvious connection still with radiology papers in the context of neuroimaging studies also involving genetics. One recent paper on the rate of incidental findings' in neuroimaging research showed that "of 1426 research imaging examinations, $567(39.8 \%)$ had at least one incidental finding (1055 total)" [13]. This is a staggering number, and the paper also went on to show that "of the 567 examinations with an incidental finding, $35(6.2 \%)$ generated clinical action, resulting in clear medical benefit in $1.1 \%$ (6 of 567) and clear medical burden in $0.5 \%$ (3 of 567)" [13]. These are small but not trivial rates of benefit and detriment resulting from the investigation of these incidental findings.

$$
\begin{gathered}
\text { "...I would suggest that the research } \\
\text { community strongly consider getting rid of } \\
\text { this term in the genomics world, and } \\
\text { instead replace it with the term 'unrelated } \\
\text { finding', 'unanticipated result' or some } \\
\text { other suitable term." }
\end{gathered}
$$

The definition of the word 'incidental' in the Merriam-Webster Dictionary is: "being likely to ensue as a chance or minor consequence, occurring merely by chance or without intention or calculation" [101]. There is therefore absolutely nothing 'incidental' about what we did, as we actively and intentionally searched for the cause of our research subject's anemia among 
the millions of nucleotides that we sequenced, as well as spending much effort trying to prove causality for these particular variants, prior to then asking the research subject's doctor to validate the results in a CLIA-certified laboratory, before returning them to the research subject. This is simply not the same as a radiologist looking at a film and immediately noticing a large mass in a place where it is not supposed to be. Radiologists spend many years perfecting their skills in pattern recognition, so that they can immediately recognize when something is dramatically wrong on a film. In fact, the human brain is exquisitely tuned for visual pattern recognition, as this skill was honed over millennia of evolution, allowing early humans to recognize very quickly when predators or other threatening things were in their immediate environment. Furthermore, radiologists typically self-select during medical school for strong visual-spatial skills, thus making it much more likely that they will be quite good at such pattern recognition tasks. Humans are simply not evolved for picking out mutations in millions or billions of nucleotides in a diploid genome, and there is simply nothing incidental about 'looking at the genomes' and somehow 'seeing' causative mutations for diseases unrelated to the research purpose.

\section{"Thus, the public and medical community will begin to understand that any result returned to a research subject must go through a rigorous confirmation of its medical actionability, so that people are not alarmed unnecessarily."}

Furthermore, there is nothing incidental or accidental about proving that a mutation actually causes a disease, either by itself (full penetrance) or in combination with other mutations or environmnent (thus, likely partial penetrance). Currently, there are many studies being published that report rare variants found more in cases versus controls or as occurring de novo in cases, but this is only correlative evidence and actually proving that these mutations by themselves cause the disease requires biological experimentation. Some authors are also defining incidental findings as "findings that are not explicitly related to the original research hypotheses (i.e., primary findings)" [14]. But, the continued use of the term 'incidental finding' threatens to trivialize, at least in the mind of the general public, the amount of time and effort that goes into determining a real 'unrelated finding.' In my informal polling of many members of the general public, their general and consistent response when asked to define 'incidental finding' is that it is something that is somehow found by accident or coincidence (which of course reflects the actual definition of the word 'incidental'). During the dozens of times in which I have consented people to participate in our genomics research, at least half or more have asked whether we will return 'any important results' to them, and this fact is certainly supported by research involving focus groups' opinions regarding return of research results [15-17]. That being said, over the past 2 years, I have never had a research subject inquire whether we will return any incidental findings' to them, and this is simply because the entire term is completely foreign and makes no sense to the general public (or to me) in this context.

\section{Conclusion}

I realize that I am fighting an uphill battle against this term 'incidental finding', given that the term is already being pervasively quoted in many genetics research papers and commentaries. Nonetheless, I would suggest that the research community strongly consider getting rid of this term in the genomics world, and instead replace it with the term 'unrelated finding', 'unanticipated result' [18] or some other suitable term. This way, we will not inadvertently continue to belittle the amount of work and effort that ought to go into validating and proving causality for any unrelated findings obtained from whole exome and/or wholegenome sequencing of human research subjects. Thus, the public and medical community will begin to understand that any result returned to a research subject must go through a rigorous confirmation of its medical actionability, so that people are not alarmed unnecessarily. This will become all the more important as whole-genome sequencing enters the clinic with the possible linking of CLIA-certified annotated genomes to medical records, as there will then be many such unrelated findings. In 20-30 years, when we will likely have a detailed catalog of human genetic variation, I can imagine that newborn babies will simply have a list of medically actionable 'findings' in their genome, allowing for proper genetic counseling and preventive measures to be implemented initially by their parents and then eventually by the growing child when they are old enough to understand their results. There is therefore nothing 'incidental' about this process. 


\section{Acknowledgements}

The author would like to thank $K$ Wang, R Robison and E Yang for helpful comments and suggestions.

\section{Financial \& competing interests disclosure}

GJ Lyon has had informal discussions with representatives from Illumina, Sage Bionetworks, ARUP, Complete Genomics and Omicia, Inc., but has not had any formal consulting role, nor has he received financial compensation from these or any other forprofit companies performing DNA collection or sequencing. The author has no other relevant affiliations or financial involvement with any organization or entity with a financial interest in or financial conflict with the subject matter or materials discussed in the manuscript apart from those disclosed.

No writing assistance was utilized in the production of this manuscript.

\section{References}

- 1 Rope AF, Wang K, Evjenth R et al. Using VAAST to identify an X-linked disorder resulting in lethality in male infants due to $\mathrm{N}$-terminal acetyltransferase deficiency. Am. J. Hum. Genet. 89(1), 28-43 (2011).

2 Lyon GJ. Personal account of the discovery of a new disease using next-generation sequencing. Interview by Natalie Harrison. Pharmacogenomics 12(11), 1519-1523 (2011).

3 Miki Y, Swensen J, Shattuck-Eidens D et al. A strong candidate for the breast and ovarian cancer susceptibility gene BRCA1. Science 266, 66-71 (1994).
4 Lyon GJ, Jiang T, Van Wijk R et al. Exome sequencing and unrelated findings in the context of complex disease research: ethical and clinical implications. Discov. Med. 12, 41-55 (2011).

Greely HT. The uneasy ethical and legal underpinnings of large-scale genomic biobanks. Annu. Rev. Genomics Hum. Genet. 8, 343-364 (2007).

6 Hayden EC. Secrets of the human genome disclosed: meeting debates ethics of revealing genetic findings. Nature 478(7367), 17 (2011).

Biesecker LG. Exome sequencing makes medical genomics a reality. Nat. Genet. 42 , 13-14 (2010).

Chen JM, Ferec C, Cooper DN. Revealing the human mutome. Clin. Genet. 78, 310-320 (2010).

Kobelka CE. Exome sequencing: expanding the genetic testing toolbox. Clin. Genet. 78, 132-134 (2010).

10 Kuhlenbaumer G, Hullmann J, Appenzellerm $S$. Novel genomic techniques open new avenues in the analysis of monogenic disorders. Hum. Mutat. 32(2), 144-151(2011).

11 Vernooij MW, Ikram MA, Tanghe HL et al. Incidental findings on brain MRI in the general population. N. Engl. J. Med. 357, 1821-1828 (2007).

12 Rodjan F, de Graaf P, Moll AC et al. Brain abnormalities on MR imaging in patients with retinoblastoma. AJNR Am. J. Neuroradiol. 31, 1385-1389 (2010).
13 Orme NM, Fletcher JG, Siddiki HA et al. Incidental findings in imaging research: evaluating incidence, benefit, and burden. Arch. Intern. Med. 170, 1525-1532 (2010).

14 Bamshad MJ, Ng SB, Bigham AW et al. Exome sequencing as a tool for Mendelian disease gene discovery. Nat. Rev. Genet. 12, 745-755 (2011).

15 Murphy J, Scott J, Kaufman D, Geller G, LeRoy L, Hudson K. Public expectations for return of results from large-cohort genetic research. Am. J. Bioeth. 8, 36-43 (2008).

16 Haga SB, Tindall G, O’Daniel JM. Public perspectives about pharmacogenetic testing and managing ancillary findings. Genet. Test. Mol. Biomarkers doi:10.1089/gtmb.2011.0118 (2011) (Epub ahead of print).

O'Daniel J, Haga SB. Public perspectives on returning genetics and genomics research results. Public Health Genomics 14, 346-355 (2011).

18 Tabor HK, Berkman, BE, Hull SC, Bamshad MJ. Genomics really gets personal: how exome and whole genome sequencing challenge the ethical framework of human genetics research. Am. J. Med. Genet. A 155A(12), 2916-2924 (2011).

\section{Website}

101 Merriam-Webster: incidental. www.merriam-webster.com/dictionary/ incidental 